\title{
Health-Related Quality of Life and Functional Outcomes from a Randomized, Controlled Study of Lisdexamfetamine Dimesylate in Children and Adolescents with Attention Deficit Hyperactivity Disorder
}

\author{
Tobias Banaschewski - César Soutullo - Michel Lecendreux • Mats Johnson • \\ Alessandro Zuddas • Paul Hodgkins • Ben Adeyi • Liza A. Squires • \\ David Coghill
}

Published online: 28 July 2013

(c) The Author(s) 2013. This article is published with open access at Springerlink.com

\begin{abstract}
Background Optimal management of attention deficit hyperactivity disorder (ADHD) aims not only to ameliorate patients' symptoms, but also to improve health-related quality of life (HRQL) and functioning. A pivotal, 7-week, randomized, double-blind, placebo-controlled, phase III study in children and adolescents in ten European countries demonstrated that the stimulant prodrug lisdexamfetamine dimesylate (LDX) is an effective and generally well-tolerated treatment for symptoms of ADHD.

Objective The aim of this study was to assess HRQL and functional impairment outcomes in this clinical trial, using the Child Health and Illness Profile-Child Edition: Parent Report Form (CHIP-CE:PRF) and the Weiss Functional
\end{abstract}

Electronic supplementary material The online version of this article (doi:10.1007/s40263-013-0095-5) contains supplementary material, which is available to authorized users.

This trial has been registered at http://www.clinicaltrials.gov (Identifier: NCT00763971).

T. Banaschewski ( $\square)$

Department of Child and Adolescent Psychiatry and Psychotherapy, Central Institute of Mental Health,

Medical Faculty Mannheim, University of Heidelberg,

P.O. Box: 1221 20, D-68072 Mannheim, Germany

e-mail: tobias.banaschewski@zi-mannheim.de

C. Soutullo

Child and Adolescent Psychiatry Unit, Department of Psychiatry and Medical Psychology, University of Navarra Clinic,

Pamplona, Spain

\section{Lecendreux}

Paediatric Sleep Centre and National Reference Centre for Orphan Diseases: Narcolepsy, Idiopathic Hypersomnia and Kleine-Levin Syndrome, Robert-Debré University Hospital, Paris, France
Impairment Rating Scale-Parent Report (WFIRS-P), respectively.

Methods Patients (aged 6-17 years) with diagnosed ADHD and a baseline ADHD Rating Scale IV total score $\geq 28$ were randomized $(1: 1: 1)$ to 7 weeks of double-blind treatment with once-daily LDX, placebo or the reference treatment, osmotic-release oral system methylphenidate (OROS-MPH). Participants' parents (or legally authorized representatives) completed the CHIP-CE:PRF and WFIRS$\mathrm{P}$ questionnaires at baseline, at weeks 4 and 7, and/or at early termination. Endpoint was defined as the last on-treatment visit with valid data ( $\leq 30 \%$ missing items). The CHIP-CE:PRF Achievement domain was pre-specified as the primary HRQL outcome.

Results The full analysis set comprised 317 patients (LDX, $n=104$; placebo, $n=106$; OROS-MPH, $n=107)$, the majority of whom completed the study (LDX, $n=77$; placebo, $n=42$; OROS-MPH, $n=72$ ). Baseline CHIP-CE:PRF $T$-scores in four of the five domains were $\geq 1$ standard deviation below norms (US

M. Johnson

Child Neuropsychiatry Unit, Queen Silvia Children's Hospital, Gothenburg, Sweden

\section{A. Zuddas}

Section of Neuroscience and Clinical Pharmacology,

Department of Biomedical Sciences,

University of Cagliari, Cagliari, Italy

P. Hodgkins $\cdot$ B. Adeyi $\cdot$ L. A. Squires

Shire Development LLC, Wayne, PA, USA

D. Coghill

Division of Neuroscience,

University of Dundee, Dundee, UK 
community samples). Compared with placebo, LDX was associated with statistically significantly improved $T$-scores from baseline to endpoint in these four domains, with effect sizes of $1.280(p<0.001)$ in Achievement, 1.079 $(p<0.001)$ in Risk Avoidance, $0.421 \quad(p<0.01)$ in Resilience and $0.365(p<0.05)$ in Satisfaction. In LDXtreated patients, placebo-adjusted improvements from baseline to endpoint in WFIRS-P scores were statistically significant $(p<0.001)$ for total score and four of the six domains, with effect sizes of 0.924 (total score), 1.249 (Learning and School), 0.730 (Family), 0.643 (Social Activities) and 0.640 (Risky Activities). OROS-MPH treatment showed similar patterns of improvement from baseline to endpoint in both CHIP-CE:PRF and WFIRS-P scores.

Conclusions Baseline HRQL and functional impairment scores reflect the burden of untreated ADHD. The benefits of short-term stimulant treatment in children and adolescents with ADHD extend beyond symptomatic relief and impact positively on HRQL and daily functioning.

\section{Introduction}

Attention deficit hyperactivity disorder (ADHD) is one of the most prevalent psychiatric disorders of childhood [1] and is increasingly recognized as a condition that can persist into adulthood [2,3]. The International Classification of Diseases, 10th Revision (ICD-10) [4], Diagnostic and Statistical Manual of Mental Disorders, 4th edition, text revision (DSM-IV-TR) [5] and DSM, 5th edition (DSM-5) [6] diagnostic criteria for ADHD all require not only the presence of symptoms, but also evidence of significant impairment in social, academic or occupational functioning. The motivation for seeking medical attention is typically the impact on an individual's behaviour in daily life in domains such as educational achievement and relations with teachers, family and peers [7]. Therefore, in addition to symptomatic relief, important goals in the treatment of ADHD are to improve health-related quality of life (HRQL) and to reduce functional impairment [8-10].

European and North American guidelines for the treatment of ADHD in children and adolescents recommend stimulants as part of a multimodal treatment plan incorporating psychoeducational, behavioural and/or other psychosocial interventions [11-18]. Lisdexamfetamine dimesylate (LDX) is the first and only stimulant prodrug. Long-acting amfetamine-based medications, including LDX, are an established front-line treatment option in North America for children, adolescents and adults with ADHD. In Europe, LDX is the only long-acting amfetamine-based medication with marketing authorization (in select countries) for the treatment of ADHD, where it is indicated in children and adolescents when response to previous methylphenidate is considered inadequate. A series of US-based, phase III clinical trials in children (aged 6-12 years) [19], adolescents (aged 13-17 years) [20] and adults (aged 18-55 years) [21] demonstrated that LDX is an effective and generally well-tolerated treatment for the symptoms of ADHD. Therapeutic benefits in simulated school or workplace environments were sustained for at least $13 \mathrm{~h}$ in children [22] and $14 \mathrm{~h}$ in adults [23] following an early-morning dose of LDX. More recently, a pivotal, double-blind, placebo-controlled phase III trial (study SPD489-325) investigated the efficacy and safety of LDX in children and adolescents with ADHD in Europe [24]. In this 7-week study, patients receiving LDX or the reference treatment (osmotic-release oral system methylphenidate; OROS-MPH) showed statistically significantly greater improvements in symptoms than those receiving placebo $(p<0.001)$, as assessed using the ADHD Rating Scale IV (ADHD-RS-IV), with effect sizes of 1.80 and 1.26 , respectively (primary efficacy outcome). On the Clinical Global Impressions-Improvement (CGI-I) scale, $78 \%$ of patients in the LDX group and $61 \%$ of those in the OROS-MPH group scored 1 ('very much improved') or 2 ('much improved'), compared with $14 \%$ in the placebo group ( $p<0.001$ for both active treatments).

We now report HRQL and functional impairment outcomes from study SPD489-325, as assessed using the Child Health and Illness Profile-Child Edition: Parent Report Form (CHIP-CE:PRF) and the Weiss Functional Impairment Rating Scale-Parent Report (WFIRS-P), respectively. The CHIP-CE:PRF is a generic, paediatric HRQL instrument with established validity and reliability in ADHD [25]. It measures overall health status, including emotional and physical well-being, behaviour, performance and involvement with family and peers. The WFIRS-P is an ADHD-specific instrument with demonstrated sensitivity $[26,27]$, and addresses domains of daily functioning that are likely to be impaired in ADHD. Assessing the impact of LDX compared with placebo on HRQL and functional impairment were pre-specified secondary objectives of the study.

\section{Methods}

The study procedures of this randomized, double-blind, parallel-group, dose-optimized, placebo-controlled, phase III trial have been described previously [24]. Study SPD489-325 (ClinicalTrials.gov identifier: NCT00763971) was conducted in accordance with current applicable regulations, the International Conference on Harmonisation of Good Clinical Practice, local ethical and legal 
requirements, and the Declaration of Helsinki (including its amendments). The study protocol was approved by an independent ethics committee/institutional review board and regulatory agency in each centre (as appropriate) before study initiation. Each patient's parent or legal guardian provided written, informed consent, and assent was obtained from each participant (as applicable) before commencing study-related procedures. The study took place between November 2008 and March 2011 [24].

\subsection{Study Population}

The study was conducted in 48 centres in ten European countries, and enrolled male and female children (aged 6-12 years) and adolescents (aged 13-17 years) who met DSM-IV-TR [5] criteria for a primary diagnosis of ADHD. Eligible patients had an investigator-rated ADHD-RS-IV total score of 28 or higher at baseline, indicating symptoms of at least moderate severity. Key exclusion criteria included failure to respond to a previous course of OROSMPH (but not of other formulations of methylphenidate) and the presence of a comorbid psychiatric diagnosis with significant symptoms (not including oppositional defiant disorder). Patients whose current ADHD medication provided effective control of symptoms with acceptable tolerability were also excluded. Enrolment was stratified such that adolescents comprised approximately $25 \%$ of the study population.

\subsection{Study Drug Administration}

Eligible patients completed a screening and washout period (up to 6 weeks) and were randomized (1:1:1) to receive once-daily LDX, placebo or the reference treatment (OROS-MPH). Randomization was stratified by country and age group (6-12 years of age or 13-17 years of age). Details of randomization and blinding have been previously described [24]. As shown in Fig. 1, the 7-week, double-blind evaluation period consisted of a 4-week doseoptimization period (visits 1-3) and a 3-week dose-maintenance period (visits 4-7), followed by a 1-week washout and safety follow-up (visit 8).

Dose optimization to LDX 30,50 or $70 \mathrm{mg}$ daily, or to OROS-MPH 18,36 or $54 \mathrm{mg}$ daily (54 mg being the maximum OROS-MPH dose approved in Europe) was based on achieving an 'acceptable response', defined as a reduction of at least $30 \%$ in ADHD-RS-IV total score from baseline and a CGI-I rating of 1 ("very much improved') or 2 ('much improved'), with tolerable side effects. Instructions were that study drugs should be taken daily upon wakening at approximately 0700 hours, beginning on the day after completion of the baseline visit (visit 0 ). Patients initially received LDX $30 \mathrm{mg}$ daily, OROSMPH $18 \mathrm{mg}$ daily or placebo. If an acceptable response was not achieved, doses were increased in $20 \mathrm{mg}$ (LDX) or $18 \mathrm{mg}$ (OROS-MPH) steps at weekly intervals. One dose reduction was permitted if a patient experienced

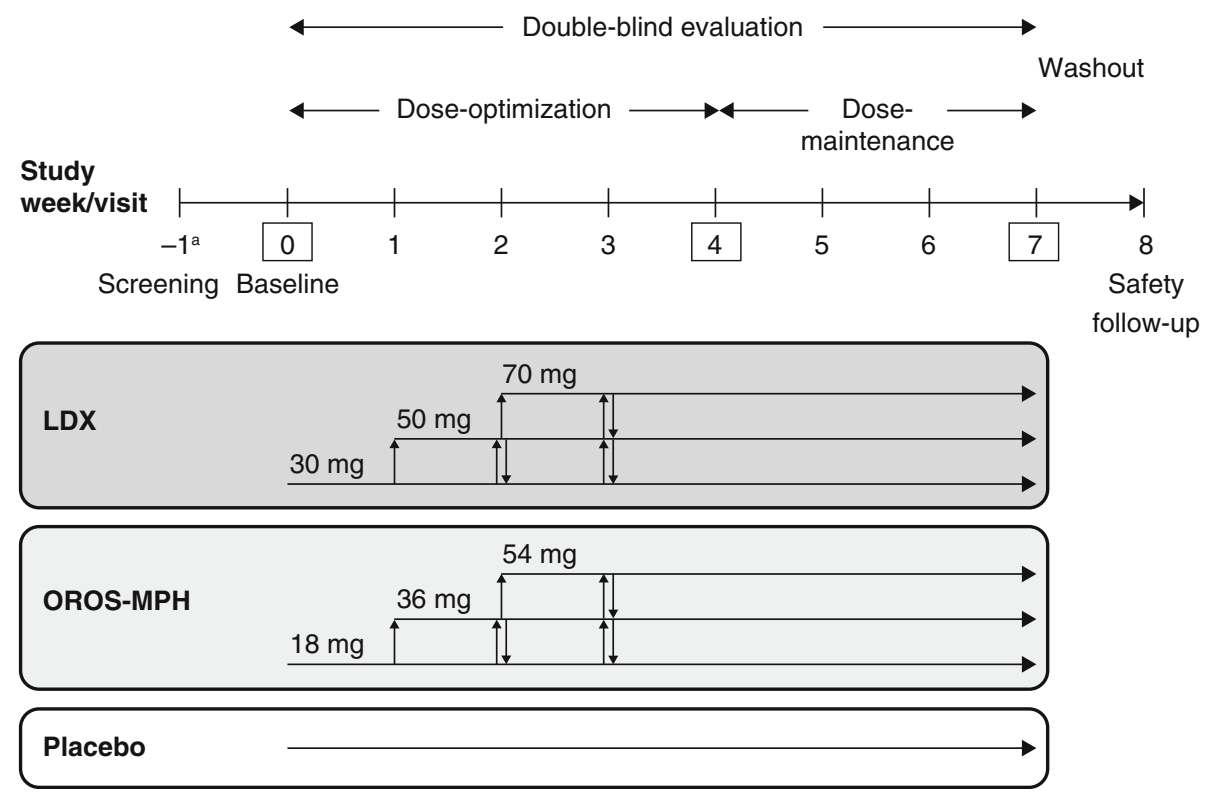

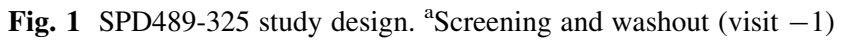
was conducted up to 6 weeks before baseline visit (week 0). At visits 1-3 (weeks 1-3), doses were titrated in the indicated single steps to achieve optimal tolerability and efficacy. From visit 4 onwards, doses could not be altered. Child Health and Illness Profile-Child Edition: Parent Report Form and Weiss Functional Impairment Rating Scale-
Parent Report assessments were performed at baseline, and then at visits 4 and 7 (shown boxed) and/or at an early termination visit attended by patients who discontinued the trial. $L D X$ lisdexamfetamine dimesylate, OROS-MPH osmotic-release oral system methylphenidate 
unacceptable tolerability. After visit 3 , the dose could not be changed: participants unable to tolerate the study drug after this point were withdrawn from the study and attended an early termination visit. Patients who achieved an acceptable response continued on their optimal dose for the remainder of the double-blind evaluation period (visits 4-7) [Fig. 1].

It should be noted that the reference arm included OROS-MPH as an active control and not as a comparator. An active control is an established treatment known to be superior to placebo. The purpose of the reference arm is to facilitate interpretation of the study, especially in the case of an apparently negative outcome in the experimental arm. The study was neither designed nor statistically powered for comparison of the test drug with the reference treatment. Although this does not preclude post hoc analysis, statistical comparisons of LDX with OROS-MPH are not presented here.

\subsection{HRQL Measure: Child Health and Illness Profile-Child Edition: Parent Report Form (CHIP-CE:PRF)}

The CHIP-CE:PRF questionnaire comprises 76 items classed into five domains and 12 associated subdomains. Most items relate to the past 4 weeks; the remainder are not associated with a specific time period. Parents use a 5-point response format to assess each item. Data were handled according to the CHIP-CE Technical Manual [28]. Domain and subdomain scores were the mean of the appropriate individual item scores (omitting items with a missing score). For a domain or subdomain score to be valid, no more than $30 \%$ of the applicable items could be missing. The domain and subdomain scores were standardized to $T$-scores with a mean of 50 and a standard deviation (SD) of 10, using an algorithm based on normative sample data from the US [29]. Higher CHIP-CE:PRF T-scores indicate better HRQL. The CHIP-CE:PRF Achievement domain was pre-specified as the primary HRQL measure.

\subsection{Functional Measure: Weiss Functional Impairment Rating Scale-Parent Report (WFIRS-P)}

The WFIRS-P questionnaire comprises 50 items grouped into six domains. The items relate to the past month and are scored using a 4-point Likert scale: 0 (never or not at all); 1 (sometimes or somewhat); 2 (often or much); or 3 (very often or very much) $[30,31]$. The WFIRS-P domain scores for each patient were the mean of the items in each domain, or of all the items for total score, omitting items with a missing or 'not applicable' score. WFIRS-P domain or total scores were considered invalid if more than $30 \%$ of the item scores used for calculation were missing or "not applicable' (with the exception of one question relating to siblings, for which a score of 'not applicable' could contribute to the minimum number of items). Higher WFIRS-P scores indicate more severe functional impairment.

\subsection{Schedule of CHIP-CE:PRF and WFIRS-P Assessments}

A parent (or legally authorized representative) completed the CHIP-CE:PRF and WFIRS-P questionnaires at baseline (visit 0), then at visits 4 and 7 (weeks 4 and 7) and/or at early termination (Fig. 1). Visit 4 (week 4) assessments were introduced via a protocol amendment after study initiation and therefore were not conducted for all participants. Endpoint was defined as the last on-treatment visit (including early termination) at which a valid CHIPCE:PRF domain or subdomain score, or valid WFIRS-P domain or total score, was obtained.

\subsection{Statistical Analyses}

All analyses were based on the full analysis set, defined as patients who were randomized and took at least one dose of study drug, but excluding patients from one site $(n=15)$ where there were violations of good clinical practice. Sample sizes were determined as previously described [24]. To assess statistically the effect of treatment with either LDX or OROS-MPH versus placebo, analysis of covariance models were applied to the change in CHIPCE:PRF domain and subdomain $T$-scores and to the change in WFIRS-P total and domain scores. The analyses included treatment group as the effect of interest (evaluated at a significance level of 0.05), the corresponding baseline score as covariate, and country and age group as blocking factors. The outputs (based on type III sum of squares) were the least-squares (LS) mean changes from baseline for each treatment group and the difference in these LS means between each drug treatment group and placebo. Effect sizes were calculated as the difference in LS means between each active drug and placebo, divided by the rootmean-square error. Conventionally, effect sizes of 0.2, 0.5 and 0.8 calculated by this method are operationally defined as small, medium and large, respectively [32]. Results were analysed using SAS ${ }^{\circledR}$ Version 9.1 .3 or higher (SAS Institute Inc., Cary, NC, USA).

\section{Results}

\subsection{Patient Disposition and Baseline Characteristics}

As described previously [24], 336 patients were randomized (LDX, $n=113$; placebo, $n=111$; OROS-MPH, 
$n=112$ ) and 196 of these completed the study. Baseline demographics and disease characteristics were similar across treatment groups [24]. The full analysis set comprised 317 patients (LDX, $n=104$; placebo, $n=106$; OROS-MPH, $n=107)$. The mean age of these patients was 10.9 years (SD 2.70 years) and $72.2 \%$ were children (aged $\leq 12$ years). The number of participants in the full analysis set who completed the trial were LDX, $n=77$; placebo, $n=42$; OROS-MPH, $n=72$. The principal reason for study discontinuation was lack of efficacy, which was reported in $48.6 \%$ of randomized patients in the placebo group, $19.6 \%$ in the OROS-MPH group and $9.7 \%$ in the LDX group [24].

\subsection{CHIP-CE:PRF}

\subsubsection{Baseline T-Scores}

At baseline, mean $T$-scores for all domains and subdomains were similar across groups (Table 1). The lowest mean domain $T$-scores at baseline were almost two population SDs (i.e. 20 points) below the normative mean (50 points), and were observed in the Achievement domain (range 30.1-31.2), which was pre-specified as the primary HRQL outcome in the study. Within the Achievement domain, mean $T$-scores were lower in the Academic Performance subdomain than in the Peer Relations subdomain. Mean $T$ scores at baseline were also below 40 (i.e. 1 SD below the normative mean) for all treatment groups in the Risk Avoidance, Resilience and Satisfaction domains. In contrast, mean Comfort domain $T$-scores were closer to the normative mean of 50. The corresponding median $T$-scores and ranges are provided in the electronic supplementary material, Table A.

\subsubsection{Placebo-Adjusted Changes at Endpoint}

In the LDX treatment group, the change in Achievement domain $T$-score from baseline to endpoint was statistically significantly greater than in the placebo group (effect size $1.280 ; p<0.001$ ) [Fig. 2]. Ranked by decreasing effect size, statistically significant improvements in $T$-scores compared with placebo were also observed in the Risk Avoidance (1.079; $p<0.001)$, Resilience $(0.421 ; p<0.01)$ and Satisfaction $(0.365 ; p<0.05)$ domains. Within these domains, there were statistically significant improvements in all subdomains except Physical Activity and Satisfaction with Self. Compared with placebo, there were no statistically significant changes in the Comfort domain or any of its subdomains in the LDX treatment group (Fig. 2).

Table 1 Mean (SD) CHIP-CE:PRF domain and subdomain $T$-scores at baseline and endpoint

\begin{tabular}{|c|c|c|c|c|c|c|}
\hline \multirow[b]{3}{*}{ Achievement } & \multicolumn{3}{|l|}{ Baseline } & \multicolumn{3}{|l|}{ Endpoint } \\
\hline & LDX & Placebo & OROS-MPH & LDX & Placebo & OROS-MPH \\
\hline & $30.9 \quad(9.71)$ & $30.1 \quad(9.37)$ & $31.2(10.19)$ & $40.3(10.54)$ & $29.3(10.06)$ & $37.7(11.20)$ \\
\hline Academic Performance & $30.3(10.18)$ & $29.4 \quad(9.24)$ & $30.5 \quad(9.28)$ & $40.7(11.24)$ & $28.4(10.89)$ & $36.9(11.25)$ \\
\hline Peer Relations & $38.6(14.56)$ & 39.2 (11.99) & $38.9(14.00)$ & $42.3(14.31)$ & $38.9(12.92)$ & $43.8(12.61)$ \\
\hline Risk Avoidance & $34.7(13.72)$ & $31.6(11.57)$ & $31.4(11.59)$ & $44.6(11.15)$ & $32.8(12.63)$ & $40.6(12.08)$ \\
\hline Individual Risk Avoidance & $41.0(15.32)$ & $40.2(12.47)$ & $37.5(13.76)$ & 49.8 (12.77) & $41.6(14.06)$ & $44.4(13.47)$ \\
\hline Threats to Achievement & $34.3(13.37)$ & $30.6(11.60)$ & $31.4(11.16)$ & $43.1(10.51)$ & $31.8(11.96)$ & $40.1(11.62)$ \\
\hline Resilience & $37.5(13.16)$ & $36.6(11.45)$ & $35.5(11.23)$ & $42.2(11.43)$ & $37.3(12.89)$ & $40.1(12.40)$ \\
\hline Family Involvement & $40.6(11.90)$ & 39.7 (11.06) & $39.6(12.03)$ & $42.8(10.78)$ & $38.2(13.13)$ & $42.3(11.72)$ \\
\hline Physical Activity & $46.0(13.04)$ & $46.4(12.33)$ & 46.7 (10.97) & $47.2(11.24)$ & $47.8(11.92)$ & $47.1(10.36)$ \\
\hline Social Problem-Solving & $38.2(13.45)$ & $36.8(12.75)$ & $34.5(12.13)$ & $44.2(12.33)$ & $38.4(13.57)$ & $40.8(14.24)$ \\
\hline Satisfaction & $35.2(14.93)$ & $34.4(14.27)$ & $36.2(13.82)$ & $39.5(14.14)$ & $36.1(13.55)$ & $40.2(12.99)$ \\
\hline Satisfaction with Health & $41.2(13.86)$ & $40.5(13.66)$ & $41.4(13.56)$ & $44.2(12.63)$ & $41.7(14.42)$ & $43.8(12.62)$ \\
\hline Satisfaction with Self & $32.3(15.75)$ & $31.5(15.58)$ & $33.9(13.70)$ & 36.7 (14.94) & $33.5(13.60)$ & $38.6(12.59)$ \\
\hline Comfort & $44.0(12.55)$ & $43.0(11.77)$ & $44.5 \quad(9.50)$ & $46.8(12.59)$ & $46.5(10.44)$ & $48.1 \quad(9.70)$ \\
\hline Physical Comfort & $50.3(10.53)$ & $50.9(10.51)$ & $52.7 \quad(8.82)$ & $52.0 \quad(9.18)$ & $53.6 \quad(8.43)$ & $53.2 \quad(9.34)$ \\
\hline Emotional Comfort & $39.3(14.11)$ & $37.2(12.47)$ & $38.4(11.31)$ & $42.7(14.90)$ & $39.7(12.55)$ & $43.0(10.83)$ \\
\hline Restricted Activity & $49.3 \quad(9.93)$ & $48.5(12.49)$ & $49.0(10.06)$ & $50.5 \quad(8.51)$ & $52.2 \quad(7.68)$ & $52.0 \quad(7.92)$ \\
\hline
\end{tabular}

Domains are shown in bold and subdomains in normal type. Higher scores indicate better health-related quality of life

CHIP-CE:PRF Child Health and Illness Profile-Child Edition: Parent Report Form, LDX lisdexamfetamine dimesylate, OROS-MPH osmoticrelease oral system methylphenidate, $S D$ standard deviation 


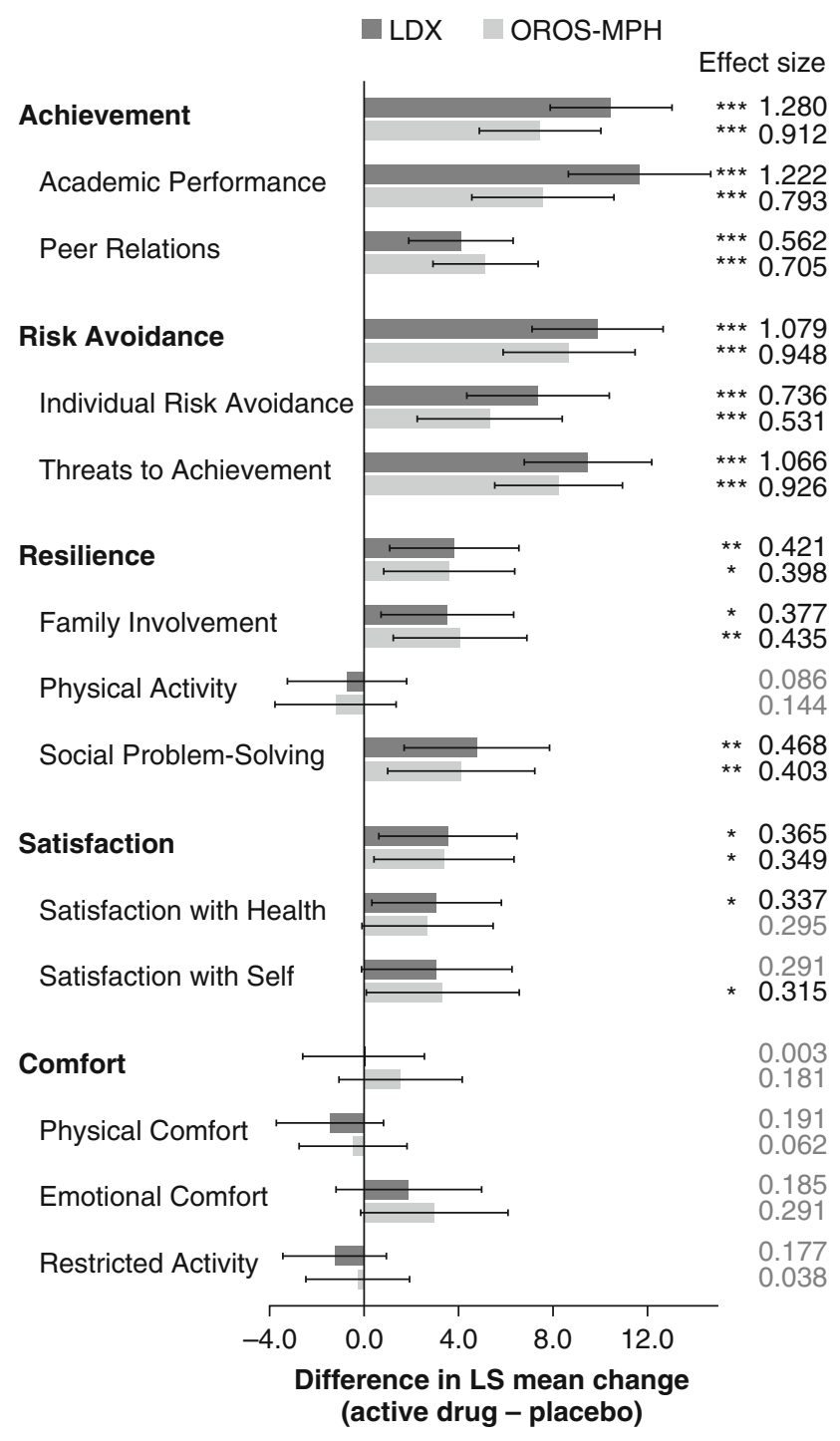

Fig. 2 Placebo-adjusted changes in CHIP-CE:PRF domain and subdomain $T$-scores from baseline to endpoint. Histogram shows the LS mean change from baseline to endpoint in CHIP-CE:PRF domain and subdomain $T$-scores for the LDX and OROS-MPH groups after subtraction of placebo values. Error bars show $95 \%$ confidence intervals. Effect size is the difference in LS mean divided by root-meansquare error. Positive differences indicate improvement compared with placebo. CHIP-CE:PRF Child Health and Illness Profile-Child Edition: Parent Report Form, $L D X$ lisdexamfetamine dimesylate, $L S$ leastsquares, OROS-MPH osmotic-release oral system methylphenidate. $* p<0.05, * * p<0.01, * * * p<0.001$ vs placebo

In the OROS-MPH reference arm, changes from baseline to endpoint were also statistically significantly greater than in the placebo group in the Achievement domain (effect size 0.912; $p<0.001$ ) [Fig. 2]. Ranked by decreasing effect size, statistically significant improvements in $T$-scores compared with placebo were also observed in the Risk Avoidance $(0.948 ; p<0.001)$, Resilience $(0.398 ; p<0.05)$ and Satisfaction (0.349; $p<0.05)$ domains. Within these domains, statistically significant improvements were observed in all subdomains except Physical Activity and Satisfaction with Health. Compared with placebo, there were no statistically significant changes in the Comfort domain or any of its subdomains in the OROS-MPH reference arm (Fig. 2).

\subsubsection{Changes from Baseline to Week 4, Week 7 and Endpoint}

In both active treatment groups, there were statistically significant improvements from baseline by week 4 in the Achievement and Risk Avoidance domains, and by week 7 in these and all other domains (Fig. 3).

\subsection{WFIRS-P}

\subsubsection{Baseline Scores}

At baseline, mean scores for each of the six WFIRS-P domains were similar across treatment groups, with the highest scores (greatest impairment) observed in the Family domain and the Learning and School domain (Table 2). The corresponding median scores and ranges are provided in the electronic supplementary material, Table B.

\subsubsection{Placebo-Adjusted Changes at Endpoint}

In the LDX treatment group, the change in WFIRS-P total score from baseline to endpoint was statistically significantly greater than in the placebo group (effect size 0.924; $p<0.001$ ) [Fig. 4]. Statistically significant improvements in scores compared with placebo were observed in the Learning and School, Family, Social Activities and Risky Activities domains $(p<0.001)$.

In the OROS-MPH reference arm, the change in WFIRS-P total score from baseline to endpoint was also statistically significantly greater than in the placebo group (effect size $0.772 ; p<0.001$ ) [Fig. 4]. Statistically significant improvements in placebo-adjusted scores were observed in the same four WFIRS-P domains as for LDX, and also in the remaining two domains, Life Skills and Child's Self-Concept $(p<0.05)$.

\subsubsection{Changes from Baseline to Week 4, Week 7 and Endpoint}

In the LDX group, statistically significant changes from baseline were observed by week 4 in WFIRS-P total score and the Learning and School, Social Activities and Risky 
Fig. 3 Changes in CHIPCE:PRF domain $T$-scores from baseline to study week 4,7 and endpoint. Circles indicate the LS mean change from baseline in $T$-scores in the indicated CHIP-CE:PRF domains for the a LDX, b OROS-MPH or c placebo groups. Error bars show $95 \%$ confidence intervals. Numbers of observations $(n)$ are shown for each data point. Positive differences indicate improvement compared with placebo. $B L$ baseline, $C H I P$ $C E: P R F$ Child Health and Illness Profile-Child Edition: Parent Report Form, EP endpoint, $L D X$

lisdexamfetamine dimesylate, $L S$ least-squares, $O R O S-M P H$ osmotic-release oral system methylphenidate, $W 4$ week 4 , $W 7$ week 7. * $p<0.05$,

$* * p<0.01, * * * p<0.001$ vs baseline

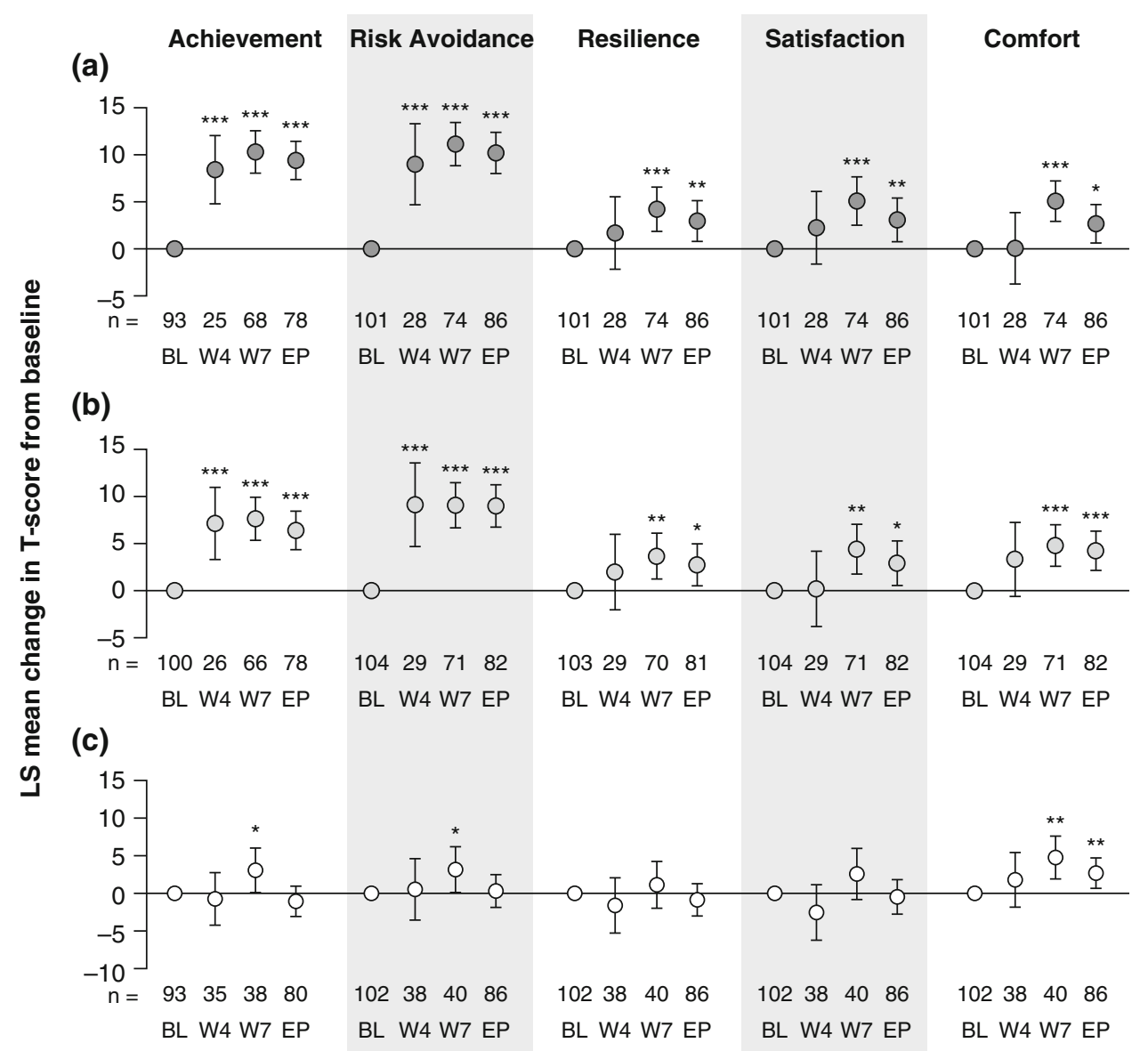

Table 2 Mean (SD) WFIRS-P domain and total scores at study baseline and endpoint

\begin{tabular}{|c|c|c|c|c|c|c|}
\hline & \multicolumn{3}{|l|}{ Baseline } & \multicolumn{3}{|l|}{ Endpoint } \\
\hline & LDX & Placebo & OROS-MPH & LDX & Placebo & OROS-MPH \\
\hline Family & $1.27(0.731)$ & $1.40(0.766)$ & $1.40(0.757)$ & $0.95(0.760)$ & $1.40(0.794)$ & $1.07(0.769)$ \\
\hline Learning and School & $1.24(0.647)$ & $1.31(0.614)$ & $1.23(0.604)$ & $0.66(0.512)$ & $1.26(0.600)$ & $0.82(0.561)$ \\
\hline Life Skills & $1.05(0.475)$ & $1.15(0.530)$ & $1.09(0.486)$ & $0.89(0.541)$ & $1.04(0.484)$ & $0.88(0.456)$ \\
\hline Child's Self-Concept & $1.05(0.878)$ & $1.09(0.781)$ & $1.00(0.747)$ & $0.83(0.840)$ & $1.00(0.825)$ & $0.73(0.757)$ \\
\hline Social Activities & $1.02(0.679)$ & $1.06(0.613)$ & $1.01(0.651)$ & $0.76(0.613)$ & $1.04(0.662)$ & $0.77(0.582)$ \\
\hline Risky Activities & $0.49(0.379)$ & $0.54(0.376)$ & $0.59(0.411)$ & $0.28(0.319)$ & $0.49(0.388)$ & $0.41(0.378)$ \\
\hline Total & $1.01(0.454)$ & $1.10(0.456)$ & $1.07(0.437)$ & $0.71(0.450)$ & $1.04(0.467)$ & $0.79(0.441)$ \\
\hline
\end{tabular}

Higher scores indicate more severe functional impairment

LDX lisdexamfetamine dimesylate, OROS-MPH osmotic-release oral system methylphenidate, $S D$ standard deviation, WFIRS-P Weiss Functional Impairment Ratings Scale-Parent Report

Activities domains, and by week 7 in these and all other domains (Fig. 5). In the OROS-MPH group, statistically significant changes from baseline were observed by week 4 in WFIRS-P total score and the Learning and School and Risky Activities domains, and by week 7 in these and all other domains (Fig. 5).

\section{Discussion}

Optimal management of ADHD aims not only to ameliorate patients' symptoms, but also to improve HRQL and to reduce functional impairment, because these are often the main motivations for seeking treatment $[7,11]$. We have 


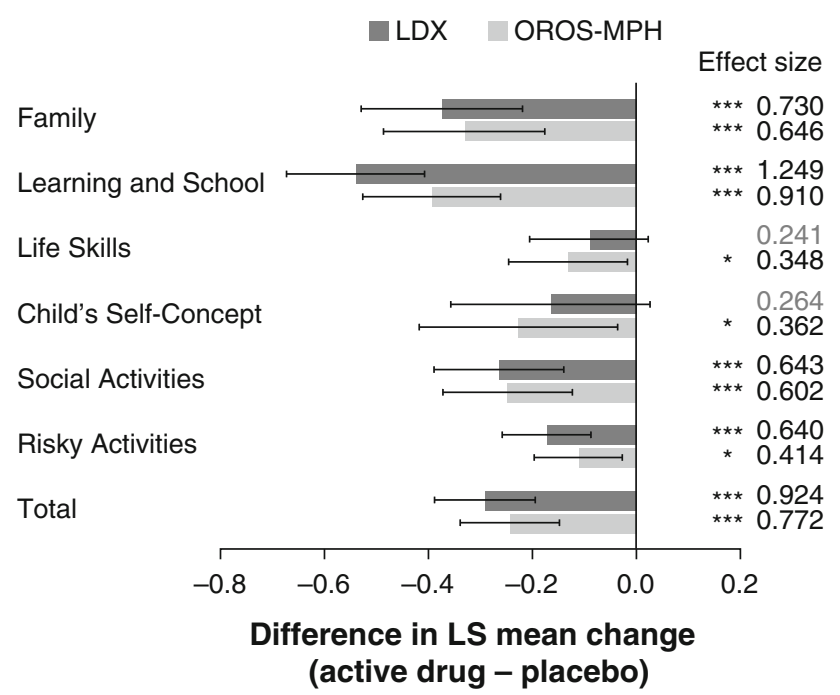

Fig. 4 Placebo-adjusted changes in WFIRS-P domain and total scores from baseline to endpoint. Histogram shows the LS mean change from baseline to endpoint in WFIRS-P domain and total scores for the LDX and OROS-MPH groups after subtraction of placebo values. Error bars show $95 \%$ confidence intervals. Effect size is the difference in LS mean divided by root-mean-square error. Negative differences indicate improvement compared with placebo. $L D X$ lisdexamfetamine dimesylate, $L S$ least-squares, OROS-MPH osmotic-release oral system methylphenidate, WFIRS-P Weiss Functional Impairment Ratings Scale-Parent Report. $* p<0.05$, $* * * p<0.001$ vs placebo

previously reported improvements in investigator-rated ADHD symptoms, measured using the ADHD-RS-IV and CGI-I instruments, in children and adolescents treated with LDX in a European, 7-week, phase III trial (SPD489-325) [24]. The present data show that LDX treatment also resulted in improvements in HRQL and functioning in this study, assessed using two parent-rated instruments, the CHIP-CE:PRF and WFIRS-P, respectively. The reference treatment, OROS-MPH, also resulted in improvements in scores on these instruments. In the CHIP-CE:PRF Achievement domain, which was pre-specified as the primary HRQL outcome, both LDX and OROS-MPH significantly improved mean $T$-scores versus placebo, with large effect sizes (1.280 and 0.912, respectively). Based on these data, the Summary of Product Characteristics, part of the European marketing authorization recently approved under the decentralized procedure, notes that LDX "showed significant improvement in child achievement in academic performance".

This is the first study of any stimulant treatment for ADHD to use the CHIP-CE:PRF. The reliability and validity of this generic, paediatric $\mathrm{HRQL}$ instrument in ADHD have been established in the prospective, panEuropean ADORE (Attention Deficit Hyperactivity Disorder Observational Research in Europe) study, which included 1477 children and adolescents with ADHD in the analysis [25], and have been confirmed in a combined analysis [33] of baseline data from 794 patients aged 6-15 years in five atomoxetine trials [33-37]. Baseline CHIP-CE:PRF values in the present study were similar to values reported in both of these analyses: mean $T$-scores in the Achievement and Risk Avoidance domains were approximately 2 population SDs (20 points) below the US community normative mean (50 points); those in the Resilience and Satisfaction domains were at least $1 \mathrm{SD}$ below the normative mean; and the mean $T$-score in the Comfort domain was within $1 \mathrm{SD}$ of the normative mean. Together, the results of these studies indicate that untreated ADHD imposes a substantial burden of illness. This is reflected by substantial economic costs of the disorder to society [38]. By comparison, in other studies using the CHIP in patient populations, mean standardized domain or subdomain $T$-scores were $1 \mathrm{SD}$ or more below the normative mean in children undergoing the last phase of maintenance therapy for leukaemia [39], but were within 1 $\mathrm{SD}$ of the normative mean in children and/or adolescents with chronic kidney disease [40-43] or asthma [44, 45].

The impact of LDX treatment was greatest in the Achievement domain of the CHIP-CE:PRF (effect size 1.280). Factor analyses of both the ADORE and combined atomoxetine study data have suggested that, unlike the subdomains of the other four CHIP-CE:PRF domains, the Academic Performance and Peer Relations subdomains of Achievement are distinct constructs in patients with ADHD $[25,33]$. This may explain the difference between the effect sizes for LDX treatment observed in these subdomains (1.222 and 0.562, respectively). A robust treatment effect of LDX was also observed in the Risk Avoidance domain (effect size 1.079). Statistically significant placeboadjusted LDX treatment effects were observed in the Resilience and Satisfaction domains, but not in the Comfort domain. At endpoint, mean $T$-scores for all CHIP-CE:PRF domains in the LDX treatment group were within approximately $1 \mathrm{SD}$ of the normative mean. A similar pattern was observed in the OROS-MPH reference arm. Thus, in both active treatment arms, the greatest impact of stimulant pharmacotherapy was observed in the domains with the most profound deficits at baseline.

Improved HRQL in LDX-treated adults with ADHD was previously documented in a 4-week, open-label study employing the ADHD Impact Module for Adults. Methylphenidate and mixed amfetamine salts have also been reported to have a beneficial impact on HRQL measured using other instruments [8, 9]. The non-stimulant atomoxetine is the only other ADHD pharmacotherapy for which the impact on HRQL has been investigated using CHIP-CE:PRF. A meta-analysis of atomoxetine studies [46] indicated that treatment benefits were most pronounced in the Achievement and Risk Avoidance domains, 


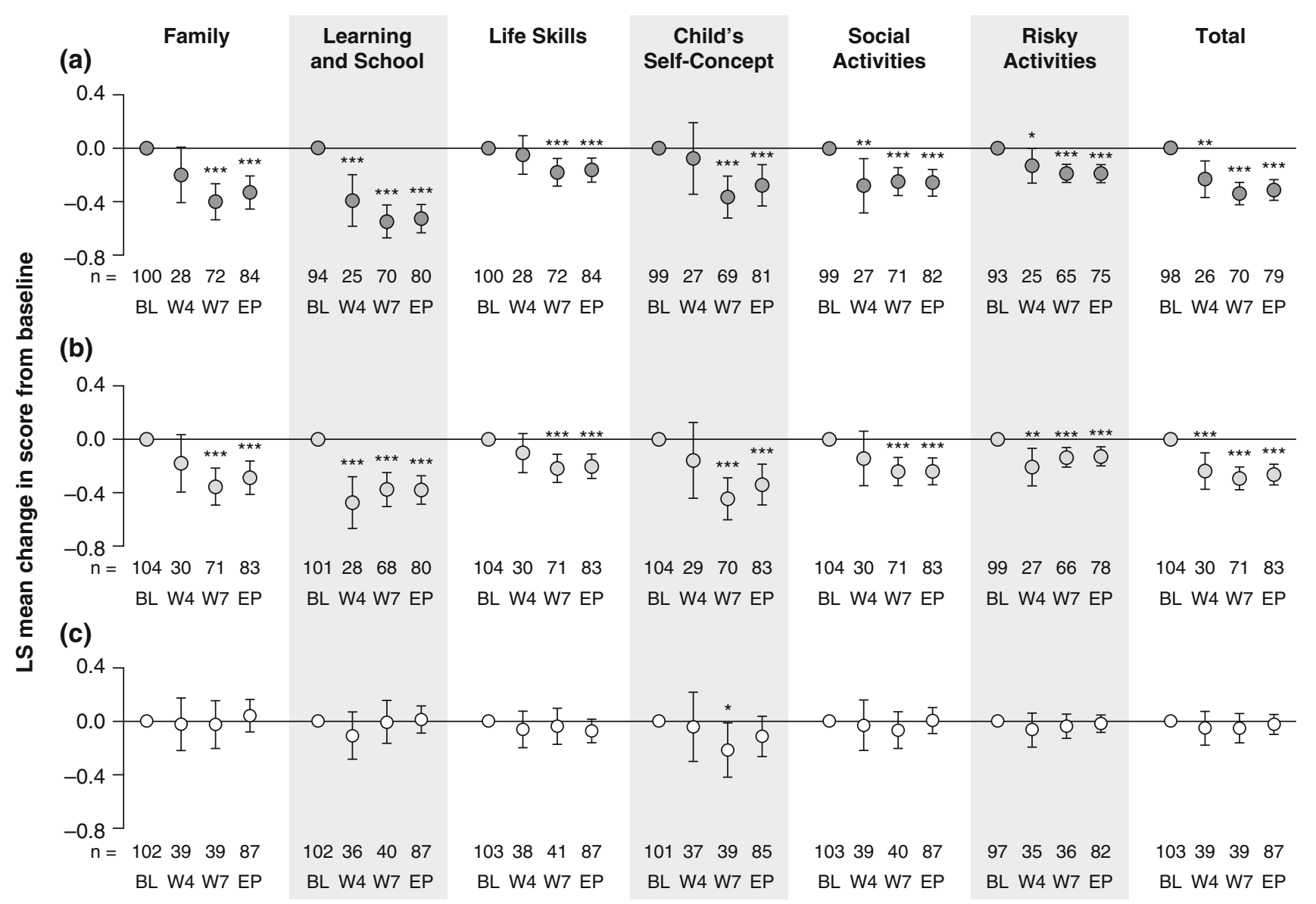

Fig. 5 Change in WFIRS-P scores from baseline to study week 4, 7 and endpoint. Circles indicate the LS mean change from baseline in scores in the indicated WFIRS-P domain or total scores for a LDX, b OROS-MPH or c placebo groups. Error bars show $95 \%$ confidence intervals. Numbers of observations $(n)$ are shown for each data point. Negative differences indicate improvement compared with placebo.

as in the present study. The effect sizes for atomoxetine in these domains (0.431 and 0.489 , respectively, [46]) were, however, somewhat smaller than those reported here for both LDX and OROS-MPH. Furthermore, unlike the stimulants investigated in the present study, atomoxetine did not statistically significantly affect scores in the Satisfaction and Resilience domains. These results suggest that earlier observations that stimulants are more effective than non-stimulants in reducing the symptoms of ADHD [47] may extend to HRQL deficits, at least as measured using the CHIP-CE:PRF.

The WFIRS-P was designed to provide a more sensitive and meaningful measure of functional impairment in patients with ADHD than that provided by generic instruments. The instrument can also be used to define impairment for clinical diagnostic purposes. As an outcome measure, it also has good internal consistency and sensitivity to change, as well as moderate convergent validity with other instruments [30]. However, published data for
$B L$ baseline, $E P$ endpoint, $L D X$ lisdexamfetamine dimesylate, $L S$ least-squares, OROS-MPH osmotic-release oral system methylphenidate, W4 week 4, W7 week 7, WFIRS-P Weiss Functional Impairment Ratings Scale-Parent Report. $* p<0.05, * * p<0.01, * * * p<0.001$ vs baseline

the WFIRS-P are limited [26, 27] and there are currently no published population norm data [30]. In the present study, mean WFIRS-P total scores at baseline were approximately 1.0 on the Likert scale (which has a range of 0-3). Although this could be interpreted as modest impairment, it is important to note that these mean responses at the domain or total level do not capture dispersion, and are likely to conceal high individual item scores of potential clinical significance. The mean total scores at baseline exceed the optimal cut-off score $(0.65)$ for differentiating children with and without ADHD, as derived by receiveroperating characteristics curve analysis in a multicentre, observational study (Hodgkins P, et al., personal communication). The effect sizes for placebo-adjusted changes from baseline to endpoint in WFIRS-P total score suggest that both active treatment effects were robust (LDX, 0.924; OROS-MPH, 0.772). As at baseline, the use of mean scores may not fully reflect treatment-induced improvements at the individual item level. In concordance with the 
CHIP-CE:PRF results, effect sizes were greatest in the WFIRS-P domains that were most impaired at baseline.

The interdependence of symptom severity, functioning and HRQL in ADHD is still uncertain and under exploration [9]. A combined analysis of atomoxetine clinical trials found that correlations between CHIP-CE:PRF $T$-scores (total, domain and subdomain) and ADHD-RS-IV total score were low (mostly below 0.4) [33]. Correlative analyses of the relevant outcome measures from the present study are awaited. However, effect sizes at endpoint were larger for ADHD-RS-IV total score (LDX, 1.80; OROSMPH, 1.26) [24] than for any domain of the CHIP-CE:PRF or WFIRS-P, supporting the notion that symptoms and HRQL or functioning are distinct, but related, constructs [48]. Furthermore, while symptomatic benefits were evident as early as study week 1 [24], some CHIP-CE:PRF and WFIRS-P domains did not show improvements until study week 7, suggesting that some aspects of HRQL and functioning may respond to stimulant medications over a longer treatment period than others. Also warranting further investigation, although modest and awaiting post hoc statistical analysis, are the differences between the two active treatments in effect sizes observed in some CHIPCE:PRF and WFIRS-P domains, which may have implications for individualized treatment [10].

The strengths of this study include its randomized, double-blind, placebo-controlled design, and the enrolment of both children and adolescents at multiple centres in Europe. Five principal limitations of the study design should, however, be considered when interpreting these results. First, the study population was enrolled according to stringent inclusion and exclusion criteria and so may not be typical of patients seen in general clinical practice. Second, dose optimization was designed to achieve balanced efficacy and tolerability, but precluded evaluation of whether the stimulant treatment effects were dose dependent and whether the LDX and OROS-MPH doses were equivalent. Third, the study was not designed or powered for direct comparison of the test drug (LDX) with the active control (OROS-MPH). Fourth, the short-term nature of the study does not permit evaluation of the long-term impact of LDX or OROS-MPH treatment on HRQL and functional outcomes. The results of SPD489-326, a followon study (with additional US patients) comprising openlabel LDX treatment for at least 26 weeks, coupled to a subsequent 6-week, double-blind, parallel-group, placebocontrolled, randomized-withdrawal period, may provide insight in this regard. Finally, the majority of patients in the LDX and OROS-MPH groups completed the study, but the majority of patients in the placebo group left the study early.

The HRQL and functional impairment instruments selected for this study also present their own strengths and weaknesses. Parental proxy assessments overcome the issue of potentially unreliable self-rating by patients with ADHD, especially younger children, but may capture different aspects of HRQL and functioning compared with self-rated instruments. For consistency, participants of all ages were assessed using the same questionnaires, despite the availability of self-rated versions of the instruments for adolescents. The CHIP-CE:PRF $T$-scores are generated using algorithms based on a US reference population, and although the Spanish CHIP-CE:PRF reference population is very similar $[49,50]$, these community samples may not be representative of normative HRQL in Europe.

\section{Conclusions}

Once-daily, optimized doses of LDX and OROS-MPH were more effective than placebo in improving HRQL and functioning in children and adolescents with at least moderately symptomatic ADHD in this European, 7-week, phase III study. The effects were seen across multiple domains of the CHIP-CE:PRF and WFIRS-P instruments, including the key domains relating to achievement in school.

Acknowledgments This study was supported by funding from Shire Pharmaceuticals. Shire develops and markets drugs to treat psychiatric disorders, including ADHD.

T. Banaschewski, D. Coghill, M. Johnson, M. Lecendreux, C. Soutullo and A. Zuddas were principal investigators in this clinical study. P. Hodgkins and L. Squires contributed to the study design. B. Adeyi was responsible for the statistical analysis. All authors were involved in discussion and interpretation of the data, critically revised the article and approved the manuscript before submission. Drs E. Southam and M. Cottingham of Oxford PharmaGenesis ${ }^{\mathrm{TM}}$ Ltd were funded by Shire to provide writing support, including editorial assistance, collation of the authors' comments and editing of the manuscript for submission.

The authors thank the patients and investigators who took part in the study.

The following authors have received compensation for serving as consultants or speakers, or they, or the institutions they work for, have received research support or royalties from the companies or organizations indicated: T. Banaschewski (Bristol-Myers Squibb, Desitin, Develco Pharma, Janssen McNeil, Lilly, Medice, Novartis, Shire, UCB and Vifor Pharma); C. Soutullo (Abbott, Alicia Koplowitz Foundation, AstraZeneca, Bristol-Myers Squibb, Carlos III Institute [Fondo de Investigación Sanitaria]: Redes Temáticas de Investigación Cooperativa, Doyma, Editorial Médica Panamericana, European Interdisciplinary Network ADHD Quality Assurance, Eli Lilly, Ediciones Universidad de Navarra, SA, Euro RSCG Life Medea, GlaxoSmithKline, Gobierno de Navarra, Grupo Aula Médica, Grupo Correo, Janssen, Medice/Juste, Novartis, Otsuka, Pfizer, University of Navarra Research projects [PIUNA], Shire, Rubiò, Solvay, Stanley Medical Research Institute, National Alliance on Mental Illness and Wolters Kluwer); M. Lecendreux (Shire, UCB and Vifor Pharma); M. Johnson (Shire, Lilly, Janssen, Novartis, Vifor Pharma and WM Lundgrens Research Fund); A. Zuddas (AstraZeneca, Bristol-Myers Squibb/Otsuka, Lilly, Lundbeck, Shire and Vifor Pharma); D. Coghill (Flynn, Janssen-Cilag, Lilly, Medice, Novartis, Otsuka, Oxford 
University Press, Pfizer, Schering-Plough, Shire, UCB and Vifor Pharma). P. Hodgkins, B. Adeyi and L. Squires are employees of Shire, and may own Shire stock and/or have stock options.

Open Access This article is distributed under the terms of the Creative Commons Attribution Noncommercial License which permits any noncommercial use, distribution, and reproduction in any medium, provided the original author(s) and the source are credited.

\section{References}

1. Polanczyk G, de Lima MS, Horta BL, Biederman J, Rohde LA. The worldwide prevalence of ADHD: a systematic review and metaregression analysis. Am J Psychiatry. 2007;164:942-8.

2. Antshel KM, Hargrave TM, Simonescu M, Kaul P, Hendricks K, Faraone SV. Advances in understanding and treating ADHD. BMC Med. 2011;9:72.

3. Stein MA. Impairment associated with adult ADHD. CNS Spectr. 2008;13:9-11.

4. World Health Organisation. International statistical classification of diseases and related health problems, ICD-10. 4th ed. Geneva: World Health Organisation; 2010.

5. American Psychiatric Association. Diagnostic and statistical manual of mental disorders, fourth edition, text revision (DSMIV-TR). Washington, DC: The Press; 2000.

6. American Psychiatric Association. Diagnostic and statistical manual of mental disorders, fifth edition (DSM-5). Arlington: American Psychiatric Publishing; 2013.

7. Parens E, Johnston J. Facts, values, and attention-deficit hyperactivity disorder (ADHD): an update on the controversies. Child Adolesc Psychiatry Ment Health. 2009;3:1.

8. Danckaerts M, Sonuga-Barke EJ, Banaschewski T, Buitelaar J, Dopfner M, Hollis C, et al. The quality of life of children with attention deficit/hyperactivity disorder: a systematic review. Eur Child Adolesc Psychiatry. 2010;19:83-105.

9. Coghill D. The impact of medications on quality of life in attention-deficit hyperactivity disorder: a systematic review. CNS Drugs. 2010;24:843-66.

10. Coghill D. Pragmatic measures in paediatric psychopharmacology-are we getting it right? Eur Neuropsychopharmacol. 2011;21:571-83.

11. American Academy of Pediatrics. ADHD: clinical practice guideline for the diagnosis, evaluation, and treatment of attention-deficit/hyperactivity disorder in children and adolescents. Pediatrics. 2011;128:1007-22.

12. Banaschewski T, Coghill D, Santosh P, Zuddas A, Asherson P, Buitelaar J, et al. Long-acting medications for the hyperkinetic disorders. A systematic review and European treatment guideline. Eur Child Adolesc Psychiatry. 2006;15:476-95.

13. Deutsche Gesellschaft für Kinder- und Jugendpsychiatrie und Psychotherapie. Hyperkinetische Störungen (F90). In: Deutsche Gesellschaft für Kinder- und Jugendpsychiatrie und Psychotherapie, editor. Leitlinien zur Diagnostik und Therapie von psychischen Störungen in Säuglings, Kindes- und Judendalter. überarbeitete Auflage ed. Cologne: Deutscher Ärtze Verlag; 2007.

14. Greenhill LL, Pliszka S, Dulcan MK, Bernet W, Arnold V, Beitchman J, et al. Practice parameter for the use of stimulant medications in the treatment of children, adolescents, and adults. J Am Acad Child Adolesc Psychiatry. 2002;41:26S-49S.

15. National Collaborating Centre for Mental Health (commissioned by the National Institute for Health \& Clinical Excellence).
Diagnosis and management of ADHD in children, young people and adults. National clinical practice guideline Number 72 . London: The British Psychological Society and The Royal College of Psychiatrists; 2009.

16. Scottish Intercollegiate Guidelines Network (SIGN). Management of attention deficit and hyperactivity disorders in children and young people - a national clinical guideline. Edinburgh: Scottish Intercollegiate Guidelines Network (SIGN); 2009.

17. Taylor E, Dopfner M, Sergeant J, Asherson P, Banaschewski T, Buitelaar J, et al. European clinical guidelines for hyperkinetic disorder-first upgrade. Eur Child Adolesc Psychiatry. 2004;13(Suppl 1):I7-30.

18. The Canadian Attention Deficit Hyperactivity Disorder Resource Alliance (CADDRA). Canadian ADHD practice guidelines. 3rd ed. Toronto: CADDRA; 2011.

19. Biederman J, Krishnan S, Zhang Y, McGough JJ, Findling RL. Efficacy and tolerability of lisdexamfetamine dimesylate (NRP104) in children with attention-deficit/hyperactivity disorder: a phase III, multicenter, randomized, double-blind, forced-dose, parallel-group study. Clin Ther. 2007;29:450-63.

20. Findling RL, Childress AC, Cutler AJ, Gasior M, Hamdani M, Ferreira-Cornwell MC, et al. Efficacy and safety of lisdexamfetamine dimesylate in adolescents with attention-deficit/hyperactivity disorder. J Am Acad Child Adolesc Psychiatry. 2011;50:395-405.

21. Adler LA, Goodman DW, Kollins SH, Weisler RH, Krishnan S, Zhang Y, et al. Double-blind, placebo-controlled study of the efficacy and safety of lisdexamfetamine dimesylate in adults with attention-deficit/hyperactivity disorder. J Clin Psychiatry. 2008;69:1364-73.

22. Wigal SB, Kollins SH, Childress AC, Squires L. A 13-hour laboratory school study of lisdexamfetamine dimesylate in school-aged children with attention-deficit/hyperactivity disorder. Child Adolesc Psychiatry Ment Health. 2009;3:17.

23. Wigal T, Brams M, Gasior M, Gao J, Squires L, Giblin J. Randomized, double-blind, placebo-controlled, crossover study of the efficacy and safety of lisdexamfetamine dimesylate in adults with attention-deficit/hyperactivity disorder: novel findings using a simulated adult workplace environment design. Behav Brain Funct. 2010;6:34.

24. Coghill D, Banaschewski T, Lecendreux M, Soutullo C, Johnson $\mathrm{M}$, Zuddas A, et al. European, randomized, phase 3 study of lisdexamfetamine dimesylate in children and adolescents with attention-deficit/hyperactivity disorder. Eur Neuropsychopharmacol. 2013; . doi:10.1016/j.euroneuro.2012.11.012.

25. Riley AW, Coghill D, Forrest CB, Lorenzo MJ, Ralston SJ, Spiel G. Validity of the health-related quality of life assessment in the ADORE study: Parent Report Form of the CHIP-Child Edition. Eur Child Adolesc Psychiatry. 2006;15(Suppl 1):I63-71.

26. Maziade M, Rouleau N, Lee B, Rogers A, Davis L, Dickson R. Atomoxetine and neuropsychological function in children with attention-deficit/hyperactivity disorder: results of a pilot study. J Child Adolesc Psychopharmacol. 2009;19:709-18.

27. Stein MA, Waldman ID, Charney E, Aryal S, Sable C, Gruber R, et al. Dose effects and comparative effectiveness of extended release dexmethylphenidate and mixed amphetamine salts. J Child Adolesc Psychopharmacol. 2011;21:581-8.

28. Riley AW, Robertson JA, Forrest CB, Green BF, Rebok GW, Starfield B. Technical Manual for the Child Health and Illness Profile-Child Edition (CHIP-CE) Parent and Child Report Forms (Version 1.0). Baltimore: Johns Hopkins University; 2001.

29. Riley AW, Forrest CB, Starfield B, Rebok GW, Robertson JA, Green BF. The Parent Report Form of the CHIP-Child Edition: reliability and validity. Med Care. 2004;42:210-20.

30. The Canadian Attention Deficit Hyperactivity Disorder Resource Alliance (CADDRA). Weiss Functional Impairment Rating 
Scale-Parent Report (WFIRS-P); 2000. http://www.caddra.ca/ cms4/pdfs/caddraGuidelines2011WFIRS_P.pdf (Accessed 18 Mar 2013).

31. The Canadian Attention Deficit Hyperactivity Disorder Resource Alliance (CADDRA). Weiss Functional Impairment Rating Scale (WFIRS) instructions; 2000 http://www.caddra.ca/cms4/pdfs/ caddraGuidelines2011WFIRSInstructions.pdf (Accessed 18 Mar 2013).

32. Cohen J. A power primer. Psychol Bull. 1992;112:155-9.

33. Schacht A, Escobar R, Wagner T, Wehmeier PM. Psychometric properties of the quality of life scale Child Health and Illness Profile-Child Edition in a combined analysis of five atomoxetine trials. Atten Defic Hyperact Disord. 2011;3:335-49.

34. Dell'Agnello G, Maschietto D, Bravaccio C, Calamoneri F, Masi G, Curatolo P, et al. Atomoxetine hydrochloride in the treatment of children and adolescents with attention-deficit/hyperactivity disorder and comorbid oppositional defiant disorder: a placebocontrolled Italian study. Eur Neuropsychopharmacol. 2009;19: 822-34.

35. Escobar R, Montoya A, Polavieja P, Cardo E, Artigas J, Hervas A, et al. Evaluation of patients' and parents' quality of life in a randomized placebo-controlled atomoxetine study in attentiondeficit/hyperactivity disorder. J Child Adolesc Psychopharmacol. 2009;19:253-63.

36. Prasad S, Harpin V, Poole L, Zeitlin H, Jamdar S, Puvanendran K. A multi-centre, randomised, open-label study of atomoxetine compared with standard current therapy in UK children and adolescents with attention-deficit/hyperactivity disorder (ADHD). Curr Med Res Opin. 2007;23:379-94.

37. Svanborg P, Thernlund G, Gustafsson PA, Hagglof B, Schacht A, Kadesjo B. Atomoxetine improves patient and family coping in attention deficit/hyperactivity disorder: a randomized, doubleblind, placebo-controlled study in Swedish children and adolescents. Eur Child Adolesc Psychiatry. 2009;18:725-35.

38. Doshi JA, Hodgkins P, Kahle J, Sikirica V, Cangelosi MJ, Setyawan $\mathrm{J}$, et al. Economic impact of childhood and adult attention-deficit/hyperactivity disorder in the United States. J Am Acad Child Adolesc Psychiatry. 2012;51(990-1002):e2.

39. San Juan AF, Chamorro-Vina C, Mate-Munoz JL, Fernandez del Valle M, Cardona C, Hernandez M, et al. Functional capacity of children with leukemia. Int J Sports Med. 2008;29:163-7.

40. Gerson AC, Riley A, Fivush BA, Pham N, Fiorenza J, Robertson $\mathrm{J}$, et al. Assessing health status and health care utilization in adolescents with chronic kidney disease. J Am Soc Nephrol. 2005;16:1427-32.

41. Dodson JL, Diener-West M, Gerson AC, Kaskel FJ, Furth SL. An assessment of health related quality of life using the child health and illness profile-adolescent edition in adolescents with chronic kidney disease due to underlying urological disorders. J Urol. 2007;178:660-5.

42. Dodson JL, Furth SL, Hsiao CJ, Diener-West M, Levey EB, Wu $\mathrm{AW}$, et al. Health related quality of life in adolescents with abnormal bladder function: an assessment using the Child Health and Illness Profile-Adolescent Edition. J Urol. 2008;180: $1846-51$.

43. Riano-Galan I, Malaga S, Rajmil L, Ariceta G, Navarro M, Loris $\mathrm{C}$, et al. Quality of life of adolescents with end-stage renal disease and kidney transplant. Pediatr Nephrol. 2009;24:1561-8.

44. Okelo SO, Wu AW, Krishnan JA, Rand CS, Skinner EA, Diette GB. Emotional quality-of-life and outcomes in adolescents with asthma. J Pediatr. 2004;145:523-9.

45. Forrest CB, Starfield B, Riley AW, Kang M. The impact of asthma on the health status of adolescents. Pediatrics. 1997;99:E1.

46. Wehmeier PM, Schacht A, Escobar R, Savill N, Harpin V. Differences between children and adolescents in treatment response to atomoxetine and the correlation between health-related quality of life and attention deficit/hyperactivity disorder core symptoms: meta-analysis of five atomoxetine trials. Child Adolesc Psychiatry Ment Health. 2010;4:30.

47. Faraone SV, Glatt SJ. A comparison of the efficacy of medications for adult attention-deficit/hyperactivity disorder using metaanalysis of effect sizes. J Clin Psychiatry. 2010;71:754-63.

48. Coghill D, Danckaerts M, Sonuga-Barke E, Sergeant J. Practitioner review: quality of life in child mental health-conceptual challenges and practical choices. J Child Psychol Psychiatry. 2009;50:544-61.

49. Estrada MD, Rajmil L, Herdman M, Serra-Sutton V, Tebe C, Alonso J, et al. Reliability and validity of the Spanish version of the Child Health and Illness Profile Child-Edition/Child Report Form (CHIP-CE/CRF). Qual Life Res. 2012;21:909-14.

50. Estrada MD, Rajmil L, Serra-Sutton V, Tebe C, Alonso J, Herdman M, et al. Reliability and validity of the Spanish version of the Child Health and Illness Profile (CHIP) Child-Edition, Parent Report Form (CHIP-CE/PRF). Health Qual Life Outcomes. 2010;8:78. 